\title{
Acute neck pain after manipulations in a healthy adolescent patient: a case report
}

\section{Background}

It is renowned that cervical spinal manipulation therapy (CSMT) is widely used among the people. It is frequently involved in the multidisciplinary treatment approach for neck pain and stiffness. These kind of treatments are often offered by many medical institutions such as non-medical institutions. Although spinal manipulation is a simple and safe therapy compared to surgical or pharmacological treatments, the serious complications caused by neck manipulation cannot be ignored. These complications include vertebral artery dissection, spinal cord or root injury, phrenic nerve injury, cervical subluxation, and cerebrovascular accidents. ${ }^{1,2}$ Cervical epidural hematoma (CEH) is an uncommon entity that can arise spontaneously or due to CSMT, cervical dislocation fractures, coagulopathies, thrombocytopenia, hemophilia, and immune diseases such as ankylosing spondylitis and Paget disease ${ }^{3,4}$ Few cases of severe quadriplegia caused by neck manipulation have been reported. ${ }^{5-15}$ Most of these cases suffered from various under-lying diseases, such as coagulation dysfunction and cervical vascular malformations. We report a case of acute CEH in a healthy adolescent male patient rapidly evolving to tetraplegia due to neck manipulations performed in the previous days. There are actually no reported cases on our knowledges of healthy adolescent patients in literature.

\section{Case description}

A 19-year-old healthy adolescent male presented to our ED in february 2020 with acute neck pain. He referred to periodically undergo cervical spine manipulations after a car accident occurred at the end of october 2019 with no reported traumatic injuries. On neurological examination he had no dysesthesia at the four extremities. Muscle power was scored as grade 5 over 5 in the upper extremities, grade 4 over 5 in the lower extremities. No deep tendon reflexes abnormalities in the extremities bilaterally. Babinski's sign and Hoffmann's signs were absent. Laboratory testing results were negligible.

Magnetic resonance imaging (MRI) with paramagnetic contrast agent of the cervical spine (Figure 1) revealed a C4-C6 extramedullary/ epidural over fluid layer occluding the posteriors perimedullary spaces. The collection was characterised by inhomogeneous signal probably linked to the presence of haemoglobin degradation products, related to the subacute evolution phase. There was an evident compressive effect on the spinal cord without signal alterations. No vessels abnormalities were found with the technique. A computed
Volume 13 Issue I - 202 I

\author{
Marco Paolo Perrini \\ University of Foggia, Italy
}

Correspondence: Marco Paolo Perrini, University of Foggia, via don lino palmisano 27 Locorotondo BA, Italy, Tel 0039328I67|727,Email perrini.marcopaol@gmail.com

Received: Febrauary 02, 202I | Published: Febrauary 12, 2021

tomographic (CT) scan (Figure 2) of the cervical spine didn't reveal fractures or other collateral findings except for the hyperdensity expressed by the extramedullary fluid collection. We leant, at the beginning, for a conservative approach keeping the patient under observation in our neurosurgery department. Three days after, the patient suddenly developed dysesthesia at the four extremities and tetraplegia with a residual capacity of slight intra rotation of the upper limbs.

A new MRI (Figure 3) in the sagittal T2 STIR sequence showed increased dimensions of the collection extending from $\mathrm{C} 7$ up to $\mathrm{C} 2$ with increased evidence of the previous compressive findings together with a new hyperintensity of the posterior cervical spinal tract linked to the medullary impairment. The patient underwent emergency $\mathrm{C} 4$ to C6 laminoplasty to evacuate this space-occupying hematoma. During the operation, an acute-subacute hematoma located in the posterior part of the spinal canal was isolated.

No vascular abnormalities were found intraoperatively.

The postoperative course was uneventful with a progressive complete recovery from the motor-sensitive deficits showed. We postoperatively performed an angiographic study, laboratory test for coagulopathies or linkable diseases without relevant results. Another MRI was performed one week after the operation emphasizing the resolution of the case (Figure 4).
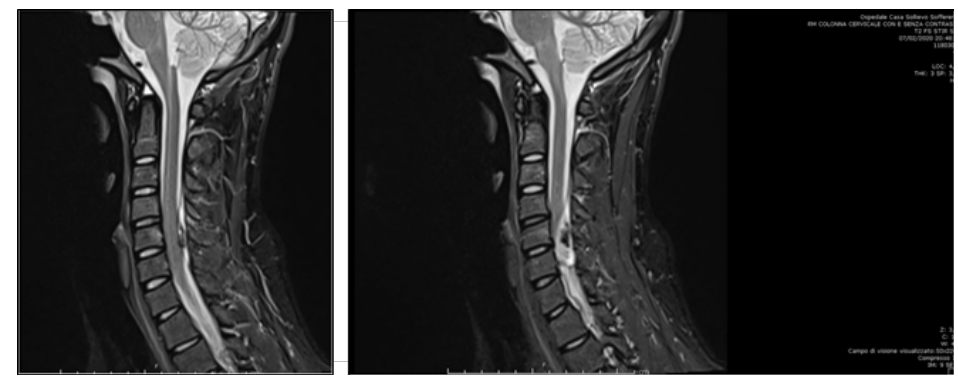

Figure I MRI with paramagnetic contrast agent of the sagittal cervical spine revealing a C4- C6 extramedullary/epidural over fluid layer occluding the posteriors perimedullary spaces. 


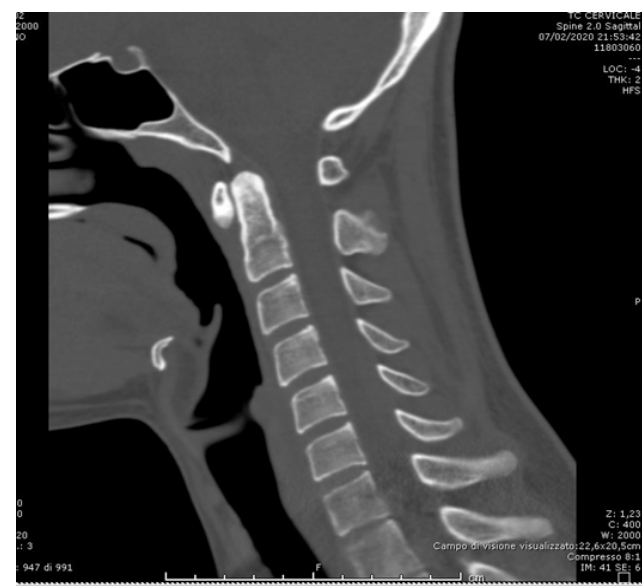

Figure 2 Sagittal CT scan view of the cervical spine underlying the hyperdensity expressed by the extramedullary fluid collection in the absence of fractures.

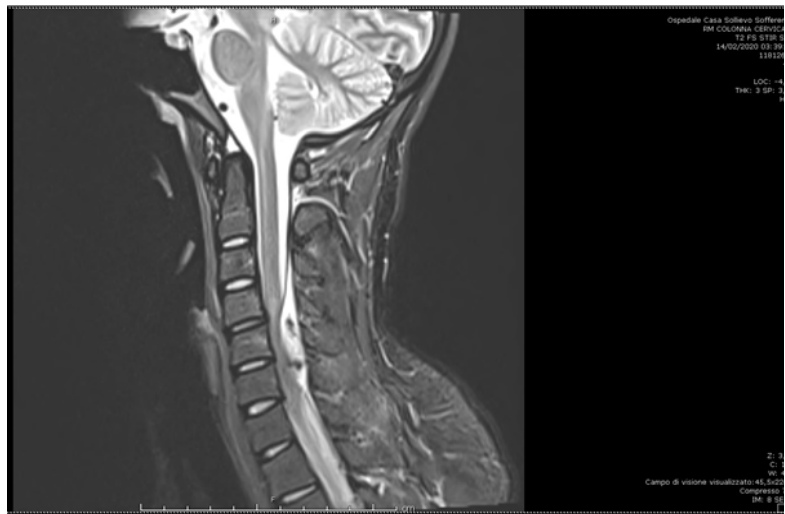

Figure $3 \mathrm{MRI}$ in the sagittal T2 STIR sequence showing increased dimensions of the collection extending from $\mathrm{C7}$ up to $\mathrm{C} 2$ with increased evidence of the previous compressive findings.

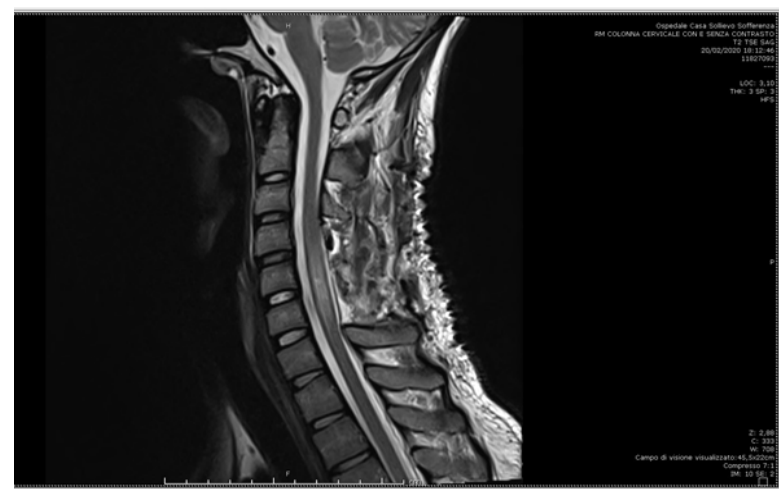

Figure $4 \mathrm{MRI}$ performed one week after the operation emphasizing the resolution of the case.

\section{Discussion}

Spinal epidural hematoma is a rare condition which may lead to serious complications.

$\mathrm{CEH}$ can arise spontaneously without an identifiable cause in $40 \%$ to $50 \%$ of reported cases or it can be caused by CSMT, cervical dislocation fractures, coagulopathies, thrombocytopenia, hemophilia, vascular malformations, neoplasm, pregnancy and immune diseases such as ankylosing spondylitis and Paget disease..$^{3,4,7,11}$ Most traumatic SEH are associated with vertebral trauma, penetrating injuries, birth trauma, or iatrogenic injuries such as epidural steroid injection, lumbar puncture, and spinal surgery. ${ }^{5}$ Cervical spinal manipulation is a common practice,used for the treatment of neck pain and stiffness, which is performed by either professional or non-professional personnel.

It is defined as: "A passive, high velocity, low amplitude thrust applied to a joint complex within its anatomical limit with the intent to restore optimal motion, function, and/ or to reduce pain". ${ }^{13}$

The incidence of complications has been estimated to be less than one injury for every 1 to 1.5 million adjustments, ${ }^{16}$ of course, there are the above mentioned factors which could expose the patient to a higher risk of bleeding complications. Other possible complications resulting from cervical spinal manipulation could include cerebral stroke from local pressure on the blood vessels, ligament injury or fractures from excessive pressure or rotation such as spinal cord injury. CEH after spinal manipulation has been reported very rarely and there are only few cases described in literature (Table 1). Before any manipulation an accurate physical examination and patient clinical history collection should always be performed.The presence of any neurological deficit on physical examination could be the signal of a CEH and should be regarded as an indicator of the patient's risk of getting severe complications during a cervical manipulation. A detailed and focused patient history collection should assess possible vasculogenic contribution, coagulopathies and conditions that might contraindicate the mobilization or manipulation. All reported CEH cases have generally occurred posteriorly or posterolaterally. ${ }^{5-15}$ The pathological mechanism of spinal epidural hematoma remains still unclear but the bleeding from the epidural vein plexus is the most widely accepted hypothesis for the mechanism of hematoma formation. ${ }^{17}$

The most probable association could be explained by the anatomical lack of valves in the epidural venous system. Any sudden rise in intrathoracic pressure, as it physiologically happens lifting a weight or coughing, may be transmitted directly to the epidural venous plexus thus causing bleeding and the formation of an epidural hematoma. ${ }^{18}$ The sudden increase in venous pressure induced by the pressure applied by the maneuvers could have led to bleeding from the epidural veins causing the formation of the hematoma in our patient. The clinical presentation of $\mathrm{CEH}$ is severe neck pain associated to the presence or absence of neurological compromission including sensory, motor, or autonomic dysfunction.

These symptoms can vary depending on the size and location of the hematoma, leading sometimes to an underestimation of the pathology in the absence of neurological deficits, anyway, almost all patients develop acute symptoms immediately after the manipulation. ${ }^{5,8}$ MRI is the gold standard imaging tool for early diagnosis of SEH. It allows to identify the lesion and its impact on the spinal cord collaterally evaluating other conditions such as herniated vertebral discs, acute bony compression, cord edema, cord contusion, and subarachnoid hemorrhage. The treatment for $\mathrm{CEH}$ depends on the severity of neurological symptoms.

Although conservative treatment has been proposed and described as a strategy to successfully treat patients with mild or no neurological symptoms, as we considered for our patient at the beginning, early 
surgical decompression is still the best choice of treatment for $\mathrm{CEH}$. In a series of 30 patients who suffered from a spinal epidural hematoma Lawton et al. examined the relationship between surgical timing and neurological outcome. The average postoperative Frankel grade decreased from 4.7 to 3.7 in patients operated on in less than six hours versus after 24 hours, and there was a corresponding decrease in rate of complete recovery from $67 \%$ to $12 \%$. Better outcomes were seen in patients operated on within 12 hours of symptom onset. ${ }^{19}$
Cervical laminectomy has been the most described surgical procedure for decompression of spinal cord in patients with $\mathrm{CEH} .{ }^{5,7,8-}$ ${ }^{10,13}$ However, standard laminectomy has also been reported to result in late cervical deformities such as kyphosis or segmental instability. ${ }^{20}$ Full recovery from all neurological deficits was reached by our patient some days after the emergency decompression surgery.

Table I Summary of reported cases of cervical epidural hematoma after spinal manipulation therapy

Reference

Age (years)

Gender Symptoms

Level

Location of hematoma Treatment

Outcome

\begin{tabular}{|c|c|c|c|c|c|c|c|}
\hline Segal et al.5 & 33 & Female & Paraplegia & C4-6 & posterior & surgery & \\
\hline Tseng et al. ${ }^{8}$ & 67 & Female & Hemiparesis & C3-5 & posterolateral & surgery & recovery \\
\hline Saxler G et al. ${ }^{6}$ & 27 & Female & Headache & $\mathrm{CI}-\mathrm{SI}$ & not reported & conservative & recovery \\
\hline Whedon et al. ${ }^{7}$ & 79 & Male & $\begin{array}{l}\text { Lower extremity } \\
\text { paralysis }\end{array}$ & $C 2-4$ & posterolateral & surgery & recovery \\
\hline Domenicucci et al. ${ }^{10}$ & 52 & Female & Hemiparesis & C4-TI & posterolateral & surgery & recovery \\
\hline Heiner et al. ${ }^{. \prime}$ & 40 & Male & $\begin{array}{l}\text { Upper extremity } \\
\text { paralysis }\end{array}$ & C4 & posterolateral & conservative & recovery \\
\hline Huang et al. ${ }^{13}$ & 40 & Male & $\begin{array}{l}\text { Upper extremity } \\
\text { paralysis }\end{array}$ & $\mathrm{C} 2-\mathrm{T} 2$ & posterolateral & Surgery & recovery \\
\hline Fattahi et al. ${ }^{14}$ & 44 & Female & Tetraplegia & $\mathrm{Cl}-4$ & anterior & Conservative & recovery \\
\hline Ling et al. ${ }^{9}$ & 33 & Male & Tetraplegia & C4-7 & posterolateral & Surgery & die \\
\hline Ryu et al. ${ }^{12}$ & 38 & Male & Paraparesis & C6-TI & anterior & Conservative & recovery \\
\hline Chen et al. ${ }^{15}$ & 55 & Male & Tetraplegia & C3-T3 & posterolateral & Surgery & $\begin{array}{l}\text { partial } \\
\text { recovery }\end{array}$ \\
\hline present case & 19 & Male & Tetraplegia & C4-C6 & posterior & Surgery & recovery \\
\hline
\end{tabular}

\section{Conclusion}

Neck pain is a common and recurrent symptom. In the absence of any neurological sign and symptom, rare pathologies such as $\mathrm{CEH}$ could be underestimate because there is no practical, clinically valid screening tests to identify underlying risks in patients with neck pain. SEH following CSMT is one of the possible, although rare, differential diagnosis to take in count in the patient history collection. Before any manipulation an accurate physical examination and patient clinical history collection should always be performed by the professional or non-professional personnel investigating for conditions that could predispose to various complications. Independently to the etiology, SEH causes severe compression of the cord, which can result in irreversible neurological deficits. The complete neurological recovery is strictly related to the time to surgical intervention. A high index of suspicion on history taking, early signs recognition, MRI evaluation and the right treatment option are crucial elements in the pathway for the best outcome. 


\section{Acknowledgments}

None.

\section{Conflicts of interest}

None.

\section{References}

1. Cassidy JD. Risk of Vertebrobasilar stroke and chiropractic care. J Manip Physiol Ther. 2009;32(2):S201-208.

2. Swait G, Finch R. What are the risks of manual treatment of the spine? Ascoping review for clinicians. Chiropr Man Therap. 2017;25:37.

3. Cox DM. Complications of cervical spine manipulation therapy:5 year retrospective study in a single-group practice. Neurosurg Focus. 2002;13(6):E1.

4. Dabbert O, Freeman DG, Weis AJ. Spinal meningeal hematoma, warfarin therapy and chiropractic adjustment. JAMA. 1970;214:2058.

5. Segal DH, Lidov MW, Camins MB. Cervical epidural hematoma after chiropractic manipulation in a healthy young woman: case report. Neurosurgery. 1996;39(5):1043-1045.

6. Saxler G, Barden B. Extensive spinal epidural hematoma-an uncommon entity following cervical chiropractic manipulation. Z Orthop Ihre Grenzgeb. 2004;142(1):79-82.

7. Whedon JM. Spinal epidural hematoma after spinal manipulative therapy in a patient undergoing anticoagulant therapy: a Case report. J Manip Physiol Ther. 2006;29(7):582-585.

8. Tseng SH. Cervical epidural hematoma after spinal manipulation therapy: case report. J Trauma. 2002;52(3):585-586.

9. Ling TH, Zakaria AFB, Abdullah ATB. Is neck massage safe? A rare case of tetraplegia and spinal shock after neck manipulation. J Orthop Surg. 2017;25(1):230949901769045.
10. Domenicucci M. Cervicothoracic epidural hematoma after chiropractic spinal manipulation therapy. J Neurosurg Spine. 2007;7(5):571-574.

11. Heiner JD. Cervical epidural hematoma after chiropractic spinal manipulation. Am J Emerg Med. 2009;27(8):1023 e1-1023.e2.

12. Ryu JI. Cervical epidural hematoma that induced sudden Paraparesis after cervical spine massage: Case report and literature review. World Neurosurgery. 2018;112:217-220.

13. Huang M. Cervical epidural hematoma after chiropractic spinal manipulation therapy in a patient with an undiagnosed cervical spinal Arteriovenous malformation. Cureus. 2015;7(8):e307.

14. Fattahi A, Taheri M. Spontaneous resolved cervical spine epidural hematoma: a case report. Surg Neurol Int. 2017;8(1):183.

15. Chen Q, Feng J, Tang X, et al. Cervical epidural hematoma after spina manipulation therapy: a case report. BMC Musculoskelet Disord. 2019;20:461

16. Powell FC, Hanigan WC, Olivero WC. A risk/benefit analysis of spinal manipulation therapy for relief of lumbar or cervical pain. Neurosurgery. 1993;33:73-8; discussion 78-79.

17. Groen RJ, Ponssen H. The spontaneous spinal epidural hematoma. A study of the etiology. J Neurol Sci. 1990;98:121-138.

18. van der Kuip M, Hoogland PV, Groen RJ. Human radicular veins: regulation of venous reflux in the absence of valves. Anat Rec. 1999;254:173-180.

19. Lawton MT, Porter RW, Heiserman JE, et al. Surgical management of spinal epidural hematoma: relationship between surgical timing and neurological outcome. J Neurosurg. 1995;83:1-7.

20. Della Pepa GM, Roselli R, La Rocca G, et al. Laminoplasty is better of laminectomy in cervical stenotic myelopathy: myth or truth? Eur Rev Med Pharmacol Sci. 2014;18:50-54. 